\title{
Umbrales de representación y proporcionalidad
}

Thresholds of representation and proportionality

Jorge Urdánoz Ganuza

Columbia University

New York - USA

ju2154@columbia.edu

Palabras clave: Umbrales de Representación, Desproporcionalidad, Representación Proporcional, Sistemas Electorales, Índices de Desproporcionalidad, Fórmulas Electorales.

\section{RESUMEN}

¿Cómo medir la proporcionalidad de los sistemas de representación proporcional (RP)? Esta cuestión se ha confundido con la de los denominados «umbrales de representación». En el artículo se repasa la historia del descubrimiento de los umbrales por parte de la Ciencia Política, intentando aclarar el fundamento conceptual de los mismos y las confusiones que los rodean. A continuación se desecha su uso como medida de proporcionalidad de los sistemas electorales y se propone para ello la idea de finura (medida por Droop). Por último, las posibles aportaciones conceptuales se recogen en una propuesta terminológica.
Keywords: Thresholds of Representation, Disproportionality, Proportional Representation, Electoral Systems, Disproportionality Indexes, Electoral Formulas.

\section{ABSTRACT}

How can the proportionality of proportional representation (PR) systems be measured? This question has been confused with the question of the so-called «thresholds of representation». This article reviews the history of the discovery of thresholds by political science, attempting to clarify their conceptual foundation and the misapprehensions surrounding them. Their valid use as an electoral system proportionality measure is ruled out, and the notion of thinness (measured by Droop) is proposed for replacing them. The article concludes with possible conceptual contributions that are brought together in a terminological proposal. 


\section{Jorge Urdánoz Ganuza}

Doctor en Filosofía por la Universidad Nacional de Educación a Distancia. Actualmente es Visiting Scholar en el Political Science Department de la Columbia University en New York (USA).

He gained his doctorate in philosophy through the Universidad Nacional de Educación a Distancia. He is currently Visiting Scholar in the Political Science Department at Columbia University in New York (USA).

Political Science Department. Columbia University. 420 West. 118 street. New York 10027. USA. 


\section{INTRODUCCIÓN}

En este artículo ofrecemos una propuesta para medir el grado de proporcionalidad de los sistemas electorales proporcionales. Tal cuestión se ha abordado por parte de la Ciencia Política desde la perspectiva de los denominados «Umbrales de Representación», una línea que históricamente desemboca en el «Umbral Efectivo» de Lijphart (1994). A nuestro juicio, ese enfoque resulta confuso y conduce a equívocos. En su lugar, ofreceremos una propuesta basada en la idea de finura, que, como intentaremos demostrar, posee, por un lado, un mayor fundamento conceptual y, por otro, resulta intuitivamente obvia, inteligible y sencilla de calcular.

Al igual que en una Nota de Investigación anterior (Urdánoz, 2006b), la perspectiva metodológica es tercamente analítica y los objetivos eminentemente conceptuales, orientados hacia la comprensión. Los «sistemas electorales» de los que hablamos aquí no tienen la misma referencia empírica que los «sistemas electorales» tal y como se entienden habitualmente en Ciencia Política. No hay distritos, ni dobles niveles, ni barreras, ni nada empírico que interfiera en la indagación teórica. Sólo hay escaños y una fórmula proporcional para repartirlos.

Hemos de reseñar aquí dos convenciones lingüísticas que adoptaremos a lo largo del artículo. Primera: cada vez que hablemos de «sistema electoral» ha de sobreentenderse una fórmula proporcional. Los sistemas mayoritarios o cualesquiera otros se excluyen del análisis, por lo que los «sistemas electorales» serán siempre, mientras no se señale lo contrario, sistemas RP. Segunda: no utilizaremos la expresión «sistemas más o menos proporcionales», sino «más o menos proporcionalistas». Siempre que venga aplicado a un sistema, el adjetivo será proporcionalismo y no proporcionalidad. El fundamento de esta convención quedará aclarado, o eso es al menos lo que esperamos, más adelante.

\section{DE ROKKAN A PENADÉS: LA BÚSQUEDA DE LOS UMBRALES}

La idea de encontrar un porcentaje que señale el número de votos necesarios para que un partido obtenga un escaño en un determinado sistema electoral es casi tan antigua como la propia idea de representación proporcional. La primera tentativa de presentar una ecuación matemática al respecto data de la segunda mitad del sigo xx y se debe a Rokkan (1968). A la misma seguirían después un buen número de contribuciones, hasta que Penadés (2000) ofrece, por fin, un conjunto de fórmulas que puede establecerse sin lugar a dudas como definitivo. Como es sabido, el umbral de votos necesarios para que un partido alcance un escaño no puede ofrecerse como un porcentaje fijo, sino más bien como un intervalo entre dos medidas: 
- Umbral Mínimo: porcentaje de votos que, bajo las circunstancias más favorables, otorga un escaño a un determinado partido. Si no lo alcanza, es imposible que logre el escaño.

- Umbral Máximo: porcentaje de votos que, bajo las circunstancias más adversas, garantiza un escaño a un determinado partido. Si lo alcanza, está garantizado que logrará el escaño.

Resulta claro que un partido cuyos votos estén entre uno y otro porcentaje puede obtener un escaño: dependerá de «las circunstancias» (es decir: de la fórmula, de cuántos partidos compitan con él y de la distribución de voto entre ellos). Además, se desprende igualmente que si no alcanza el Umbral Mínimo se queda sin escaño y que si alcanza el Umbral Máximo consigue escaño con seguridad. Por supuesto, aunque venimos hablando de lograr «un» escaño, la investigación ha perseguido desde sus orígenes (Rae et al., 1971) descubrir el valor de ambos umbrales no sólo para uno, sino para cualquier número de escaños (por ejemplo: ¿qué porcentaje de votos necesita un partido para alcanzar 4 escaños?). Se trata, entonces, de hallar los Umbrales Mínimo y Máximo para cualquier número E de escaños de entre los M que están en juego¹.

La historia de la búsqueda de estos umbrales por parte de la Ciencia Política se ha descrito de varias formas (Taagapera, 1998: 414; Penadés, 2000: 126-130; Lin, 2003: 51; RuizRufino, 2005b: 91-92). A nuestro juicio, los principales jalones de la misma pueden presentarse, muy brevemente, divididos en dos grupos. Por un lado, las aportaciones de los siguientes autores:

1 El Umbral Mínimo suele denominarse «de Representación» o «de Inclusión». El Umbral Máximo se conoce igualmente como «de Exclusión». Penadés utiliza además otras expresiones, ya que se ocupa de más aspectos: «Funciones de Pago», «Funciones de Umbral», de «Votos suficientes y necesarios» o de «Votos mínimos y máximos» (Penadés, 2000). Nosotros utilizaremos tan sólo las expresiones Umbral Mínimo y Máximo. Si no especificamos E, entonces se sobreentiende que E=1. 


$\begin{array}{cccc} & \text { Ste. } & \text { Ste. Laguë } & \text { Restos } \\ \text { D'Hondt } & \text { Laguë } & \text { Modificado } & \text { Mayores }\end{array}$

Rokkan (1968)

- Umbral Mínimo

$\frac{V^{\prime}-1}{M+P-1} \quad \frac{V-1}{(2 M+P-2)}$

$\frac{\mathrm{V}}{\mathrm{MP}}$

Rae et al. (1971)

- Umbral Máximo
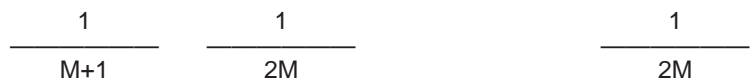

- Umbral Mínimo
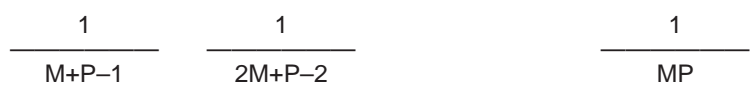

Lijphart y Gibberd (1977)

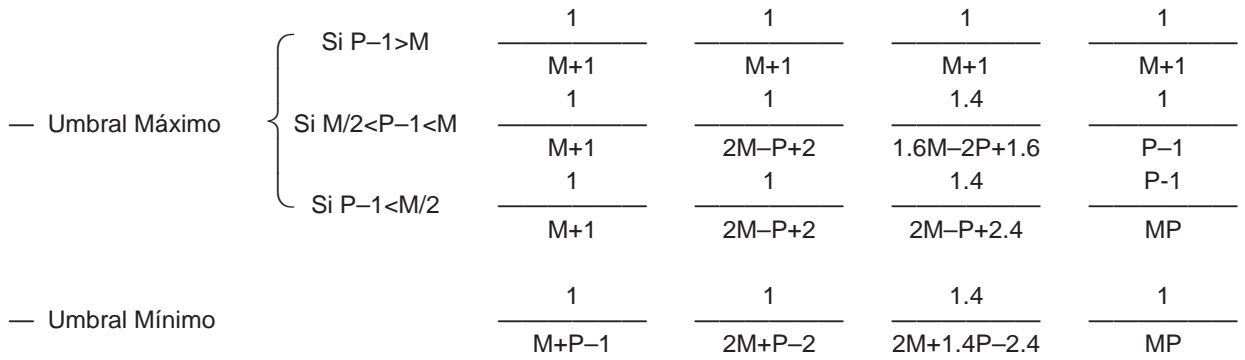

M: Número de escaños que se eligen en el sistema.

$P$ : Número de partidos.

E: Número de escaños a conseguir para los que se calcula el Umbral (si no aparece, E = 1).

V: Número de votos.

Por otro, Gallagher (1992), en quien se advierte muy positivamente la influencia de Balinsky y Young, ofrece los umbrales para nueve fórmulas:

Si $M>(P-1)$

Imperiali Divisor

$2 /(M+3)$

D'Hondt

VUT

$1 /(M+1)$

$1 /(\mathrm{M}+1)$

Ste. Lagüe Modificado

$1,4 /(2 \mathrm{M}-\mathrm{P}+2.4)$

$1 /(2 \mathrm{M}-\mathrm{P}+2)$

te. Lagüe

$1 /(3 \mathrm{M}-2 \mathrm{P}+3)$

$1 /(\mathrm{M}+1)$

Imperiali Restos más altos

$1 /(\mathrm{M}+1)$

Droop

$(\mathrm{P}-1) / \mathrm{PM}$

$2 /(\mathrm{M}+2 \mathrm{P}-1)$

$1 /(\mathrm{M}+\mathrm{P}-1)$

$1.4 /(2 \mathrm{M}+1.4 \mathrm{P}-2.4)$

$1 /(2 \mathrm{M}+\mathrm{P}-2)$

$1 /(3 \mathrm{M}+\mathrm{P}-3)$

$3 /(\mathrm{P}(\mathrm{M}+2)$

$2 /(\mathrm{P}(\mathrm{M}+1)$

RM Hare

$1 / \mathrm{PM}$ 
A partir de este estado de cosas, Penadés (2000) alcanza en su obra una comprensión global y unificada de todas las fórmulas electorales y ofrece una sola función generatriz para calcular los umbrales de las mismas. Con objeto de introducir tal función generatriz recurriremos a la siguiente nomenclatura para las fórmulas:

FÓRMULAS DE DIVISOR

\begin{tabular}{|c|c|}
\hline \multirow[t]{3}{*}{ (Adams) } & Precio Máximo (0) \\
\hline & Precio 0.1 \\
\hline & Precio 0.2 \\
\hline \multirow[t]{2}{*}{ (Danés) } & Precio 0.3 \\
\hline & Precio 0.4 \\
\hline (Ste. Lagüe, Webster) & Precio 0.5 \\
\hline (Dos tercios) & Precio 0.6 \\
\hline & Precio 0.7 \\
\hline & Precio 0.8 \\
\hline & Precio 0.9 \\
\hline (D'Hondt, Jefferson) & Precio Mínimo (1) \\
\hline
\end{tabular}

FÓRMULAS DE CUOTA

\begin{tabular}{r|l} 
& \\
Cuota-3 & \\
Cuota-2 & \\
Cuota-1 & \\
Precio Natural (0) & (Hare RM, Hamilton) \\
Cuota+1 & (Droop) \\
Cuota+2 & (Imperiali) \\
Cuota +3 & (Imperiali Reforzada) \\
&
\end{tabular}

Frente a la nomenclatura clásica o tradicional (la que aparece en las dos columnas de los extremos), la notación presentada en las dos columnas centrales puede caracterizarse de científica o sistemática, en cuanto que permite iluminar las propiedades esenciales de cada fórmula, en especial su funcionamiento y su relación con el sesgo². Más allá de eso, lo que nos interesa ahora es que cada fórmula de divisor viene caracterizada por un decimal y cada fórmula de cuota por un modificador de la cuota, como puede verse. Identificando las fórmulas mediante tales características numéricas, Penadés ofrece las ecuaciones mediante las que es posible hallar los umbrales de todas las fórmulas proporcionales ${ }^{3}$ :

\footnotetext{
2 Hemos defendido las ventajas de esta nomenclatura (que Penadés ya adelanta en su obra de 2000) en Urdánoz (2007). Además de unívoca e iluminativa, esta terminología resulta informativa. Con sólo referirnos a una fórmula podemos saber: a) qué grado de sesgo la caracteriza (mayor cuanto más alejada esté la fórmula de 0.5 o de la Cuota Natural y menor cuanto más cerca), y b) a qué partidos beneficia (si es menor de 0.5 o aumenta la cuota, beneficiará a los pequeños; si es mayor o la reduce, a los grandes). Así, por ejemplo, las fórmulas «0.8» y «0.2» tienen idéntico sesgo, aunque una beneficia a los grandes y otra a los pequeños: lo sabemos con sólo nombrarlas. Lo mismo ocurre con «cuota+2» y «cuota-2».

3 Dado cualquier E, en las ecuaciones para el Umbral Máximo se ha de calcular E-1 (es decir: si queremos calcular el número máximo de votos para que un partido consiga 1 escaño, calcularemos en la ecuación que E=0 escaños; si queremos el porcentaje de votos para 7 escaños, calcularemos $E=6$, etc.).
} 
Umbral Máximo

Si $E<M-P+1$

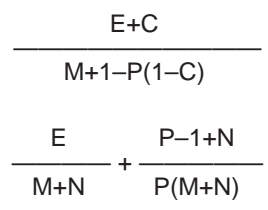

Si $\mathrm{E}>\mathrm{M}-\mathrm{P}+1$

\begin{tabular}{lccc} 
FÓRMULAS DE DIVISOR & $\frac{E+C}{M+1-P(1-C)}$ & $\frac{E+C}{(M+1) C+E(1-C)}$ & $\frac{E-1+C}{M-1+P C}$ \\
\cline { 2 - 3 } FÓRMULAS DE CUOTA & $\frac{E}{M+N}+\frac{P-1+N}{P(M+N)}$ & $\frac{E}{M+N}+\frac{M-E+N}{(M-E+1)(M+N)}$ & $\frac{E-1}{M+N}+\frac{1+N}{P(M+N)}$
\end{tabular}

Umbral Mínimo

C: Decimal que aparece en el nombre de la fórmula.

$\mathrm{N}$ : Número que modifica la cuota en el nombre de la fórmula.

Gracias a las mismas, dado cualquier sistema electoral es posible calcular siempre ambos umbrales (conociendo la fórmula, el número de partidos y el número de escaños). Por ejemplo, con la fórmula Precio Mínimo y cuatro partidos compitiendo por 12 escaños, podemos predecir que si el partido A desea obtener 5 escaños, su Umbral Mínimo es de $33,33 \%$ y su Umbral Máximo de 38,47\%. Las ecuaciones que acabamos de exponer para los umbrales (junto con sus pertinentes demostraciones) vienen así a culminar toda una línea de investigación que acompaña al estudio de los sistemas electorales prácticamente desde sus inicios, y en la que han participado buena parte de los principales representantes de la literatura electoral (Rae et al., 1971; Grofman, 1975; Lijphart, 1986; Taagepera y Shugart, 1989; Gallagher, 1992; entre otros ${ }^{4}$ ).

\section{UMBRALES Y PROPORCIONALIDAD}

Aunque es obvio que los umbrales de representación revisten un enorme interés desde un punto de vista teórico, cuestión sobre la que enseguida volveremos, lo que nos interesa en este artículo es sobre todo el hecho de que los mismos pueden entenderse de una manera un tanto confusa, en especial si se utilizan como indicadores de la proporcionalidad de los sistemas electorales (es decir, cuando se asume algo así como que «a menor umbral de representación, más proporcionalista es un sistema electoral»). En los apartados que siguen intentaremos desvelar las contradicciones de este enfoque.

\footnotetext{
4 La obra de Penadés supone por ello un evidente avance conceptual en el terreno de la sistemática electoral. Es el primero, que sepamos, en ofrecer el cálculo para los umbrales de todas las fórmulas, no sólo para los de las proporcionales, las únicas que nos interesan aquí, sino también para los de las mayoritarias y para los de las que él denomina igualitarias —también Palomares y Ramírez, casi inmediatamente después de él (Palomares y Ramírez, 2003, entregado en 2001), alcanzan el cálculo para los umbrales de las fórmulas proporcionales-. Además de ello, si la obra de Penadés se hubiera traducido al inglés, probablemente se hubieran adelantado muchas aportaciones que vieron la luz en el ámbito internacional con posterioridad y que ya en su obra quedaban sobradamente respondidas (Marshall, Olkin y Pukelsheim, 2002; Schuster, Pukelsheim, Drton y Draper, 2003; Lin, 2003; Schwingenschlogl y Drton, 2004). Véase Penadés (2000 y 2007).
} 


\subsection{Hipótesis: los umbrales como indicador del proporcionalismo}

¿Qué factores influyen para que un determinado sistema electoral sea más o menos proporcionalista? En primer lugar y sin duda alguna, el número de escaños. Aunque más adelante profundizaremos en esta relación, es obvio que a mayor $\mathrm{M}$, más proporcionalista será el sistema. Es fácil ver que un sistema con 50 escaños es más proporcionalista que uno con 3. Esta evidencia, sin embargo, no cancela la cuestión, puesto que la fórmula también marca una diferencia: dos sistemas con $\mathrm{M}$ idéntico pero distinta fórmula no pueden ser igualmente proporcionalistas, uno lo será más que el otro. Así, probablemente la afirmación de que un sistema con 10 escaños y fórmula Precio Mínimo es «menos proporcionalista» que otro con 10 escaños y fórmula Precio Medio encontraría algo muy cercano al consenso entre los especialistas en Ciencia Política (Benoit, 2000). A partir de ahí aparecen casos difíciles: imaginemos, por ejemplo, dos sistemas, uno con 10 escaños y Precio Mínimo y otro con 9 escaños (por tanto, «menos proporcionalista» según M) pero con Precio Medio ( $y$, por tanto, «más proporcionalista» de acuerdo a la fórmula). ¿Cuál de los dos es «más proporcionalista»? El cálculo de los umbrales puede ofrecer una respuesta cuantitativa y, por tanto, definitiva. Para el ejemplo anterior, los umbrales máximos respectivos son 9,09 y 5,56\%. Así, puede concluirse que el segundo sistema es «más proporcionalista».

Este uso de los umbrales está presente desde el principio de la investigación (Loosemore y Hanby, 1971) a lo largo de casi cuatro décadas (Grofman, 2001:300), teniendo su resultado más conocido en la variable del Umbral Efectivo de Lijphart (1994). A nuestro juicio, sin embargo, resulta desacertado utilizar así los umbrales: los mismos no señalan el proporcionalismo de cada sistema, sino el número de votos que un determinado partido (y sólo uno) necesita para alcanzar un escaño en ese sistema, lo cual es considerablemente diferente.

\subsection{Una refutación}

En la siguiente tabla hemos calculado los diferentes Umbrales Máximos (los porcentajes que señalan los votos a superar para obtener con seguridad un escaño) para diferentes sistemas electorales. Los sistemas son diferentes porque se modifican la fórmula y $\mathrm{M}$, pero en todos los casos suponemos que compiten dos partidos. Obtenemos: 
FÓRMULA

PRECIO MÁXIMO

PRECIO 0.1

PRECIO 0.2

PRECIO 0.333

PRECIO 0.4

PRECIO MEDIO

CUOTA NATURAL $\}$

PRECIO 0.666

PRECIO 0.7

PRECIO 0.8

PRECIO 0.9

PRECIO MÍNIMO

\begin{tabular}{|c|c|c|c|c|c|c|c|c|c|c|c|c|c|c|}
\hline \multicolumn{15}{|c|}{$M=$} \\
\hline 2 & 3 & 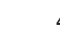 & 5 & 5 & 6 & 7 & 8 & 9 & 10 & 20 & 50 & 100 & 200 & 1000 \\
\hline 0 & 0 & ( & c & 0 & 0 & 0 & & 0 & 0 & 0 & 0 & 0 & & \\
\hline 8,33 & 4,55 & 3,1 & 2,38 & &, 92 & 1,61 & 1,39 & 1,22 & 1,09 & 0,52 & 0,20 & 0,10 & 0,05 & 0,01 \\
\hline 14,29 & 8,33 & 5,88 & 4,55 & & 3,70 & 3,13 & 2,70 & 2,38 & 2,13 & 1,03 & 0,40 & 0,20 & 0,10 & 0,02 \\
\hline 20,00 & 12,50 & 9,0 & 7,14 & & 5,88 & 5,00 & 4,35 & 3,85 & 3,45 & 1,69 & 0,67 & 0,33 & 0,17 & 0,03 \\
\hline 22,22 & 14,29 & 10,5 & 8,33 & &, 90 & 5,88 & 5,13 & 4,55 & 4,08 & 2,02 & 0,80 & 0,40 & 0,20 & 0,04 \\
\hline 25,00 & 16,67 & 12,5 & 10,00 & & 3,33 & 7,14 & 6,25 & 5,56 & 5,00 & 2,50 & 1,00 & 0,50 & 0,25 & 0,05 \\
\hline 28,57 & 20,00 & 15,3 & 12,50 & & ,53 & 9,09 & 8,00 & 7,14 & 6,45 & 3,28 & 1,32 & 0,66 & 0,33 & 0,07 \\
\hline 29,17 & 20,59 & 15,9 & 12,96 & & , 94 & 9,46 & 8,33 & 7,45 & 6,73 & 3,43 & 1,39 & 0,70 & 0,35 & 0,07 \\
\hline 30,7 & 22,22 & 17,3 & 14,25 & & 2,12 & 10,53 & 9,30 & 8,33 & 7,55 & 3,88 & 1,58 & 0,80 & 0,40 & 0,08 \\
\hline & 3,68 & 18,7 & 15,5 & & 3,24 & 11,54 & 10,23 & 9,18 & 8,33 & 4,33 & 1,77 & 0,89 & 0,45 & 0,09 \\
\hline 33,33 & 25,00 & 20,0 & 16,67 & & 1,29 & 12,50 & 11,11 & 10,00 & 9,09 & 4,76 & 1,96 & 0,99 & 0,50 & 0,10 \\
\hline
\end{tabular}

¿Señalan tales porcentajes un orden de los sistemas en relación al ideal de proporcionalidad? No, en absoluto. Dados dos sistemas, uno con $\mathrm{M}=10$ y otro con $\mathrm{M}=3$, si el primer sistema aplica Precio Mínimo y el segundo Precio 0.1, entonces para un partido pequeño será mucho más fácil conseguir representación en el segundo sistema (sólo necesitará un $4,55 \%$ de los votos) que en el primero (que le exige un 9,09\%). Pero de ahí no se desprende que el segundo sistema sea «más proporcionalista». De hecho, sólo hemos de olvidarnos de las cifras (que a veces no nos dejan ver el bosque) y pensar con un poco de sentido común cómo funciona tal sistema: si sólo hay dos partidos y 3 escaños y el partido pequeño logra 1 escaño con un 4,55\% de los votos, entonces el partido grande, con un 95,45\% de los votos, sólo ha obtenido 2 escaños: todo parece indicar que no estamos ante un sistema demasiado proporcionalista. Por el contrario, el otro sistema está exigiendo un 9,09\% para alcanzar un escaño de los 10 que se ofrecen, lo cual parece sin duda alguna más razonable.

Una cosa es el umbral que un partido ha de superar para lograr un escaño y otra cosa una medida que señale el proporcionalismo del sistema. El umbral está calculado bajo una hipótesis (que establece cuántos partidos compiten) y bajo la perspectiva de un partido concreto (el pequeño). Extrapolar tal porcentaje al sistema en sí no tiene ninguna justificación: ni la hipótesis tiene por qué cumplirse ni la perspectiva ha de ser la de ese partido pequeño.

Debería resultar suficiente esgrimir la reductio ab absurdum que permiten los umbrales de la fórmula Precio Máximo: siempre equivalen a 0, lo que supone que 1 voto garantiza siempre un escaño (lo cual es escrupulosamente cierto bajo tal fórmula). Si se asume que los umbrales señalan el proporcionalismo del sistema, entonces todo sistema que funcione con tal fórmula (incluso aunque $\mathrm{M}=1$, es decir, aunque sea un sistema «mayoritario») ha de 
catalogarse automáticamente como el más proporcionalista, lo cual no resulta muy congruente.

\subsection{El uso de los umbrales}

Que los umbrales no hayan de interpretarse en clave de proporcionalismo del sistema no significa que carezcan de utilidad, por descontado. El principal provecho que los umbrales pueden depararnos es eminentemente teórico y está relacionado con el progreso de la comprensión, por parte de la Ciencia Política, de las características de las diferentes fórmulas electorales. En palabras de Penadés: "calcular las funciones generatrices de las funciones de umbrales de todas las fórmulas electorales (...) aclara, de modo que juzgo definitivo, algunas cuestiones importantes sobre el funcionamiento de las fórmulas. Creo que al poder verlas "todas juntas", me atrevo a decir que por primera vez, resultan inmediatas las semejanzas entre unas y otras, su parentesco con la proporcionalidad y, sobre todo, su parentesco con la fórmula mayoritaria» (Penadés, 2000: 1225).

Además de otros posibles usos teóricos, cuya gama puede ser bastante amplia y fructífera (Ruiz-Rufino, 2005a), se ha de señalar un uso específicamente práctico de los umbrales: el que viene dado por su aplicación predictiva. Los umbrales deberían interesar a quienes, como los dirigentes partidistas o las empresas demoscópicas, hayan de realizar previsiones sobre posibles aventuras escisionistas o sobre futuros resultados electorales. Este uso, sin embargo, al estar supeditado a una realidad empírica específica, y sólo a ésa, se torna más desdibujado que el anterior (además de resultar considerablemente menos interesante desde un punto de vista científico, desde luego). Los umbrales no son, al fin y al cabo, más que una generalización basada en los dos extremos posibles para cualquier partido: las circunstancias «más adversas» y las «más favorables». Sobra decir que para delimitar la situación concreta en la que se encuentra un determinado partido la predicción teórica de ambos extremos tendrá una utilidad siempre menor que la observación empírica directa, ad hoc, de su situación real (siempre que se cuente con los adecuados instrumentos para acotar con precisión esta última, por supuesto).

Más allá de todo ello, si desechamos los umbrales como instrumento de medición del proporcionalismo, seguimos enfrentados al problema que se intentaba solucionar: ¿cómo introducir la incidencia de la fórmula en el proporcionalismo del sistema?

5 Penadés es perfectamente consciente, como no puede ser de otra forma, de la clase de malentendido que estamos denunciando aquí, y no atribuye en ningún momento a sus umbrales categoría de índice de proporcionalidad o similar (Penadés, 2000: 132). 


\section{FINURA Y PROPORCIONALIDAD}

\subsection{La idea de finura}

De manera un tanto elemental, puede afirmarse que todo sistema RP será más proporcional cuanto más fino sea. Un sistema de reparto es más fino cuanto menor es la unidad mediante la que se lleva a cabo el reparto. Si una empresa paga a sus trabajadores utilizando únicamente billetes de 500 euros, estamos ante un sistema de reparto muy grueso y es de esperar que los empleados no reciban exactamente (proporcionalmente) lo que merecen. Es probable que quien debía cobrar 3.100 euros reciba 3.000 , y que quien había trabajado lo correspondiente a 1.800 euros reciba 2.000. Unos se verán perjudicados, otros beneficiados. Todo esto no ocurre debido a que el sistema monetario es muy fino y, por tanto, muy proporcionalista: la entidad dinero puede dividirse en fracciones muy pequeñas (céntimos), de manera que puede repartirse en proporción exacta al trabajo realizado.

Una primera medida obvia de la proporcionalidad de un sistema RP sería así la fracción $1 / \mathrm{M}$, que atrapa esta idea, repetimos que muy elemental, de finura. En nuestro ejemplo, esa fracción para la entidad «dinero» era de 500 euros (muy gruesa) en el primer caso y de 1 céntimo (muy fina) en el segundo. La entidad «representación» puede dividirse igualmente de modo más o menos fino, dependiendo del número de escaños. Si sólo existen cinco escaños, cinco «billetes» de un $20 \%$ de la representación, es probable que el reparto de la misma entre los partidos dé lugar a perjudicados y a beneficiados. Si los escaños son 100, sin embargo, la representación se repartirá mediante «billetes» de un 1\% y los partidos podrán recibir una proporción mucho más ajustada.

Esta idea de finura se desdibuja cuando introducimos las posibilidades que abren las diferentes circunstancias que caracterizarán inevitablemente a cualquier reparto concreto: el número de partidos y la fórmula. Dado un sistema con $\mathrm{M}=10$, podemos decir que la finura es del 10\%, pero, a la vez, sabemos que si la fórmula es Precio Medio, entonces a un partido le bastará lograr un 5\% de los votos para alcanzar un escaño; mientras que si la fórmula es Precio Mínimo, entonces necesitará un 9,09\%. Y eso si sólo hay dos partidos. Si los partidos son tres, entonces nuestro partido requerirá un 4,76 y un $8,33 \%$, respectivamente. Con cuatro partidos tendremos otros porcentajes, etc. Uno puede concluir entonces que la finura del $10 \%$ tiene, al fin y al cabo, poca o ninguna importancia. Por supuesto, hemos vuelto a introducir, primero, la cuestión de los umbrales y de las fórmulas (que son dos caras de la misma moneda) y, segundo, la del número de partidos. Nos ocuparemos en primer lugar y muy brevemente de esta última.

Inicialmente, podría pensarse que el número de partidos debería tenerse en cuenta, puesto que su incidencia es obvia. Pero introducir esa variable hace imposible configurar cualquier 
medida de proporcionalidad para sistemas electorales mínimamente formal o, si se prefiere, universalizable. En primer lugar, eso interferiría en la labor predictiva: buscamos un índice de proporcionalidad del sistema electoral (variable institucional) que nos permita elaborar hipótesis con respecto a la influencia del mismo en el sistema de partidos (realidad empírica); pero si en el mismo cálculo del índice recogemos ya el número de partidos, entonces, por decirlo del algún modo, estamos poniendo el carro delante de los bueyes. Además, y en segundo lugar, introducir el número de partidos vulneraría también la coherencia comparativa de la variable: no podríamos comparar sistemas electorales con diferente número de partidos, dado que obviamente cuantos más partidos haya, más reducido será el índice ${ }^{6}$.

Esto parece claro, pero la fórmula sigue marcando una diferencia. Sin referencia alguna al número de partidos, queremos averiguar qué sistema es más proporcional para $M=10$, uno con Precio Mínimo u otro con Precio Medio. ¿Podemos hacerlo? Para contestar a esa cuestión partiremos de la afirmación (Urdánoz, 2006a) que establece la existencia de dos grandes concepciones de la proporcionalidad: la Global (que se relacionaría con las fórmulas de cuota) y la Binaria ${ }^{7}$ (que lo haría con las fórmulas de divisor). Tales dos grandes concepciones pueden describirse como sigue.

La concepción Global de la proporcionalidad es la más intuitiva de las dos, la que más se acerca a la noción que habitualmente poseemos sobre lo que significa «proporcional». Desde esta perspectiva la proporcionalidad es una relación que se establece entre las partes y el todo, de tal manera que, como suele decirse, cada parte recibe lo que le corresponde de ese todo. Resulta obvio que cada una de las partes sólo adquiere sentido con relación a una globalidad, a un conjunto a partir del cual ella misma aparece definida como «parte», siendo así que sin tal conjunto la cuestión misma de la proporcionalidad parecería no tener significado alguno ni, por tanto, sería posible plantearla.

Frente a ello, la concepción Binaria de la proporcionalidad asume un enfoque diferente. Desde la misma no es en absoluto necesario recurrir a un conjunto o una globalidad dividida en partes: la cuestión se resuelve acudiendo a la relación que mantienen los diferentes protagonistas del reparto comparándose los unos con los otros en atención a sus propios méritos, sin alusión a un todo. La proporcionalidad no se entiende aquí como una relación entre las partes y el todo, sino como una propiedad establecida únicamente entre los diversos partidos entre sí.

\footnotetext{
6 De hecho, sea cual sea el sistema (con cualquier valor para M y cualquier fórmula proporcional), puede ocurrir que un partido con dos votos tenga asegurado siempre un escaño. Sólo hay que suponer que es el partido más votado, y que tras él vienen miles de partidos con un solo voto. Incluso podría suceder que tal sistema fuera «mayoritario», si M=1, si bien el índice de proporcionalidad lo describiría, de incluir en su cálculo el número de partidos, como extraordinariamente proporcionalista.

7 En Urdánoz (2006a), la obra en la que introducíamos esta distinción, denominamos «Parcial» a la concepción que aquí vamos a designar «Binaria». Las razones del cambio terminológico se expondrán enseguida.
} 


\subsection{Proporcionalidad y fórmulas de divisor}

Una consecuencia de esta distinción es la siguiente: si nos mantenemos fieles a la concepción de la proporcionalidad Binaria (la de las fórmulas de divisor), entonces ni siquiera hay un «sistema» tal y como se está entendiendo aquí (en la medida en que nos encontramos buscando un Índice de proporcionalismo «del sistema»). La mera idea y el concepto mismo de «sistema» sólo pueden concebirse desde los presupuestos de la proporcionalidad Global. Intentaremos explicar esto.

EI DRAE define la voz sistema como «conjunto de cosas que relacionadas entre sí ordenadamente contribuyen a un determinado objeto». Tenemos, entonces, tres aspectos necesarios para hablar de un sistema: ciertas cosas, una relación ordenada entre ellas y un objeto (un todo, una globalidad) al que vienen referidas. Esta definición de sistema la encaja perfectamente, como un guante, la concepción Global de la proporcionalidad: tenemos los partidos (que son las cosas, las «partes» en el sentido que el DRAE recoge como «porción de un todo»), tenemos una relación ordenada entre ellas (la fórmula) y tenemos un objeto al que vienen referidas (el todo, el total de los escaños): se trata así de dar a cada parte su cuota proporcional de ese todo.

Sin embargo, las fórmulas de divisor funcionan de una manera tal que no existe el tercer elemento: no hay una globalidad, un todo, un objeto al que vengan referidas las partes, sino sólo partidos y una relación entre ellos. Aquí los partidos pueden también considerarse «partes», pero no en el sentido anterior («porción de un todo»), sino más bien en el que también recoge el DRAE de «cada uno de los ejércitos, facciones, sectas, banderías, etc., que se oponen, luchan o contienden» (si bien, por descontado, sin la connotación militarista que destila tal definición). Un partido no tiene aquí «un 23\%», «un 43\%» o «un 19\%» de «los» votos. Para ese cálculo es necesario un total de votos que conformen el $100 \%$, pero en este contexto no existe tal cosa. Cada partido sólo tiene una cierta cantidad de fuerza, sus votos, y con ellos compite con la fuerza, los votos, de los otros partidos. Cada combate es binario, un partido contra otro. La fórmula marca la relación entre las partes, de acuerdo a los escaños ya ganados. No hay «un total», no hay una «globalidad», no hay «un 100\%» ni de votos ni de escaños a repartir. Aquí, sencillamente, no existen las variables «V»y «M». Tampoco existe $\mathrm{P}$, el número de partidos: por muchos que haya, en cada combate sólo existen dos, ni uno más ${ }^{8}$.

8 Cuando introdujimos la distinción (Urdánoz, 2006a) adoptamos la denominación «proporcionalidad Parcial», debido a que la segunda acepción del DRAE que hemos recogido nos permitía considerar a los partidos políticos como «partes» pero evitando la idea de «porción de un todo». Con todo, ahora somos firmes partidarios de su sustitución por «proporcionalidad Binaria». En primer lugar, tal etiqueta terminológica recoge mucho más certeramente la naturaleza matemática de esta concepción de la proporcionalidad, en la que sólo existen enfrentamientos a dos. Además, también la segunda acepción de DRAE está 
Así que cuando hablamos de «un sistema con Precio Mínimo, $\mathrm{M}=10$ y $\mathrm{P}=3$ », por ejemplo, y nos preguntamos por «su índice» de proporcionalidad, lo que ocurre en realidad es que le estamos endilgando a la concepción Binaria de la proporcionalidad cuestiones propias de la Global que no puede responder más que contradictoriamente. No hay «un sistema», sino sólo combates binarios, a dos, y por tanto no puede haber un índice de algo que no existe. Sólo hay, si se quiere, pequeños y sucesivos «microsistemas» (en cada uno de los cuales tenemos dos partidos, cada uno con sus respectivos votos y escaños ya ganados, y un escaño en juego) regulados por la relación que imponga la fórmula. No parece posible establecer «el índice» de un mecanismo así y extrapolarlo al «sistema».

De hecho, los umbrales de las fórmulas de divisor no son sino la transposición al lenguaje de la proporcionalidad Global (y en concreto al lenguaje del porcentaje, que es la «finura» que usamos en la vida cotidiana para dividir prácticamente cualquier entidad) de una confrontación a dos, y no pueden ser otra cosa, pues no otra cosa hacen las fórmulas de divisor. Es cierto que pueden calcularse tales umbrales para sistemas «de varios partidos». Pero, a la vez, no lo es. En realidad, si calculamos los umbrales para un «sistema con cinco partidos», en el fondo lo que estamos haciendo es únicamente calcular un combate a dos, entre el grande y el pequeño, utilizando las posibilidades que ofrecen los otros tres partidos para dibujar o bien las «peores» circunstancias o bien las «más halagüeñas» para el partido pequeño. Es decir, dicho de otra manera, dado que sabemos que hay otros tres partidos, calculamos las dos posibilidades extremas desde la perspectiva del pequeño: que influyan de la manera más perniciosa en el combate a dos o bien que influyan de la manera más benéfica. Sólo después transformamos los resultados de ambas confrontaciones a dos (de ambos microsistemas binarios con tres partidos invitados, por así decir) en dos porcentajes, el Umbral Máximo y el Mínimo, y podemos deducir que, si hay cinco partidos, entonces si un partido alcanza el porcentaje Mínimo puede que gane en el enfrentamiento y que, si alcanza el Máximo, gana tal enfrentamiento con seguridad.

Es fácil ver que al operar así hemos de partir no del sistema en sí, sino más bien de un determinado partido inmerso en un determinado enfrentamiento binario. Sólo después de establecer tal enfrentamiento añadimos las necesidades que «el sistema» impone (en nuestro caso, tres partidos comparsa). Por ello, el porcentaje del umbral será sólo aplicable a tal

teñida, ella misma, de elementos de la proporcionalidad Global: los ejércitos, facciones, sectas y banderías son, al fin y al cabo, partes de un todo (un todo que es una batalla o un enfrentamiento). «Parte» viene obviamente de «partir», y por tanto remite inevitablemente a un todo anterior a la partición o, al menos, un todo al cual la partición viene referida. Pero para la proporcionalidad Binaria no existe ese todo o, si se quiere, el único «todo» que le es dado concebir es siempre binario: dos partidos. Así, la proporcionalidad Global puede concebir los partidos como diferentes partes de un todo que es la sociedad (el sistema), pero para la proporcionalidad Binaria no existe tal sociedad (no hay, como venimos diciendo, sistema alguno), sino siempre y necesariamente tan sólo dos partidos. Obviamente, cuando sólo existen dos partidos, ambas perspectivas coinciden (como lo hacen, en tal caso, las fórmulas de divisor y las de cuota: Penadés, 2000: 16). 
partido (porque es el protagonista del enfrentamiento que hemos supuesto para calcular tal umbral), pero no al sistema en sí (que sólo viene, aquí, a ejercer de decorado del enfrentamiento: un decorado tan necesario como subsidiario).

Esta última idea puede expresarse también así: sólo mediante la idea de umbral (aplicable únicamente a un partido pero no a un sistema) pueden introducirse los rasgos diferenciales que caracterizan a las distintas fórmulas de divisor. Porque con las fórmulas de divisor es obligado partir de un partido concreto. Si no, sencillamente, no es posible proceder: no tenemos dónde aplicar la propia noción de proporcionalidad. El problema es, claro, que si partimos de un partido en concreto, desembocamos no en un índice (del sistema), sino más bien en un umbral (de un determinado partido). Y, utilizados como indicadores de la desproporcionalidad, los umbrales nos conducen a la clase de extravagancias denunciadas en el apartado 3.2.

\subsection{Proporcionalidad y fórmulas de cuota}

Como sabemos, la concepción de la proporcionalidad de la que parten las fórmulas de cuota es la Global, por lo que encajan perfectamente la noción de sistema. Pero, más allá de eso, no hemos de olvidar que también los umbrales de las fórmulas de cuota están concebidos necesariamente desde la perspectiva de un determinado partido, y no son por tanto una propiedad del sistema en sí. Podemos verificarlo mediante los umbrales de la fórmula Cuota Natural, la más sencilla e intuitiva de todas, que serían éstos (calculados para dos partidos):

FÓRMULA

\begin{tabular}{rrrrrrrrrrrrrr}
\multicolumn{11}{c}{$M=$} \\
\hline 2 & 3 & 4 & 5 & 6 & 7 & 8 & 9 & 10 & 20 & 50 & 100 & 200 & 1000 \\
25,00 & 16,67 & 12,50 & 10,00 & 8,33 & 7,14 & 6,25 & 5,56 & 5,00 & 2,50 & 1,00 & 0,50 & 0,25 & 0,05
\end{tabular}

Es inmediato entender el sentido de tales medidas y el modo en que están subordinadas a la perspectiva de un partido. Si sólo hay dos partidos y dos escaños a repartir, entonces si un partido tiene un 25\% (más uno, como suele añadirse) de los votos, obviamente se lleva un escaño. Si aseveramos entonces que el sistema es «proporcional al 25\%», o algo así, con la misma e idéntica legitimidad podríamos aseverar que lo es «al 75\%» si nos fijáramos en la perspectiva del otro partido. Los umbrales indican muy bien el sesgo (de la fórmula), pero no tanto la proporcionalidad del sistema. 


\subsection{Sesgo y proporcionalidad}

Los dos sistemas que hemos mencionando antes (ambos con $\mathrm{M}=10$ pero uno con Precio Medio y otro con Precio Mínimo) se diferencian en la fórmula y, por tanto, son «sistemas diferentes». Ahora bien, ¿implica eso que uno ha de ser más proporcionalista que el otro? ¿Es inevitable enfocar así la diferencia que los separa? La respuesta que estamos defendiendo aquí establecería que los umbrales no sirven como indicadores de un mayor o menor proporcionalismo, sino que únicamente señalan el sesgo: si la fórmula de un sistema beneficia más a los pequeños que el otro (o viceversa) o, lo que es lo mismo, si un sistema está más sesgado que otro hacia los pequeños (o hacia los grandes).

Precisamente porque los umbrales se relacionan indefectiblemente con un partido, y sólo con él, únicamente pueden indicarnos el sesgo, pero no el proporcionalismo. Los umbrales máximos para nuestros dos sistemas son 9,09 y 5\%. Como sabemos, tales porcentajes aseguran que un partido obtendrá escaño bajo tal sistema. Ahora bien, tales umbrales están construidos bajo la hipótesis de dos partidos y se refieren sólo al pequeño. Si queremos hacer justicia al «sistema» habremos de acudir igualmente a los porcentajes señalados para el partido grande. Tales porcentajes señalan que el partido grande necesita, respectivamente, un 90,91 y $95 \%$ de votos para conseguir 9 escaños. Por tanto, para cada escaño se requieren un $10,1 \%$ de los votos en el primer sistema y un 10,55\% en el segundo. Así, desde la perspectiva del grande las tornas, lógicamente, se han modificado: si confundimos sesgo con proporcionalidad, entonces deberíamos deducir ahora que, de los dos sistemas, es «más proporcionalista» el primero (Precio Mínimo), cuando antes lo era el segundo (Precio Medio).

En consecuencia, aunque la búsqueda de los umbrales se haya confundido con la cuestión del proporcionalismo de los sistemas, difícilmente pueden éstos interpretarse así. Los umbrales no tienen que ver con «el sistema» sino de manera secundaria y relegada, luego sólo de modo contradictorio pueden reflejar su proporcionalismo. Lo que reflejan es, sin duda, el sesgo de las diferentes fórmulas proporcionales, lo cual es algo considerablemente diferente ${ }^{9}$.

9 Las fórmulas, los sistemas y los resultados son todos elementos clave para la comprensión de la noción de proporcionalidad que comparten un mismo peligro conceptual: la confusión entre sesgo y proporcionalidad. En esta Nota de Investigación nos ocupamos de la cuestión desde la perspectiva de los umbrales y los sistemas, sin entrar, más que secundariamente, en la cuestión de las fórmulas (de los resultados ya nos hemos ocupado en Urdánoz, 2006b). 


\subsection{Retomamos la finura}

Estamos buscando un índice de proporcionalismo para sistemas electorales, una medida que dictamine qué sistema de entre dos o más sistemas electorales es «más proporcional». Ahora bien: ¿qué significa «más proporcional» en tal oración? A nuestro juicio, el sentido de tal expresión en ese contexto surge de la concepción Global de la proporcionalidad. De hecho, el arraigo intuitivo de tal noción es tan manifiesto que, por así decirlo, no es tanto que nosotros adoptemos tal noción de proporcionalidad, sino más bien que es ella la que nos escoge, la que nos ha escogido antes incluso de plantear la investigación.

El mero hecho, en primer lugar, de buscar un índice representable como un porcentaje de un total (un $\mathrm{x} \%$ ) es un enfoque que obedece a tal noción y depende por completo de ella. Si nuestra visión del mundo funcionara con la proporcionalidad Binaria nos hubiera bastado con la «proporción» que se impone en cada enfrentamiento a dos. Hubiéramos elegido una de tales proporciones sin mayores contratiempos. No buscaríamos (ni nosotros ni todos los autores que se han ocupado del tema durante décadas) un $\mathrm{x} \%$, sino algo como «dos veces mayor» 0 «0,7 veces menor», o algo así, lo que no es el caso en absoluto.

Pero, además, no sólo la idea de porcentaje está teñida de la noción Global, sino que también lo está igualmente el propio concepto de «sistema», como ya hemos visto. La concepción Binaria de la proporcionalidad no puede concebir «sistemas electorales», sino tan sólo enfrentamientos binarios. Partir de la idea de sistema supone ya, de por sí, iniciar la investigación bajo la égida de la proporcionalidad Global. Las fórmulas de divisor son, cierto, técnicas de reparto efectivas, pero la concepción de la proporcionalidad que las constituye nos resulta por completo insólita y extraña. Aunque podamos con ellas proceder a un reparto, los objetivos del mismo, los valores que lo conforman normativamente y, sobre todo, los términos que utilizamos para entender tal reparto nacen, toman cuerpo y se nutren de la proporcionalidad Global.

Como resulta obvio, la noción de finura que hemos introducido surge del paradigma de la proporcionalidad Global: si un sistema tiene 10 escaños, su finura es de un 10\% (la proporcionalidad funcionará por lotes de $10 \%$, por así decirlo). Cuando manejamos esa idea nos estamos basando en la noción de proporcionalidad más obvia y evidente, la que dicta el sentido común, la Global. Este sencillo procedimiento lógico no podemos, sin embargo, siquiera concebirlo desde la proporcionalidad Binaria: el mismo planteamiento (buscar un índice de proporcionalidad «del sistema electoral») no tiene sentido desde sus presupuestos. Si formulamos así el objetivo se debe a que nos encontramos presos de las nociones y conceptos propios de la proporcionalidad Global (que es la intuitiva y cotidiana) y los trasladamos, inevitable e inconscientemente, a la proporcionalidad Binaria, que no funciona con ellos y se mueve en unos parámetros por completo diferentes. 
Lo que hemos establecido hasta ahora es que, dado un sistema electoral, gracias a $\mathrm{M}$ podremos establecer su finura, o cuán proporcionalista es (cuánta cantidad de proporcionalidad lo caracteriza, aunque suene un poco raro decirlo así). La fórmula nos transmitirá cómo pone en juego ese sistema su finura: si a favor de los grandes o de los pequeños (sesgo). Si mediante el uso de los umbrales mezclamos ambas cosas, el resultado será inconsistente.

Se trata así de no mezclar sesgo con proporcionalidad. Un sistema en el que se reparten 5 escaños es más proporcional que un sistema en el que se reparten 4. La única razón es que es más fino. Diferentes fórmulas harán que cada sistema juegue con su finura de un modo o de otro (que beneficie más a los grandes, más a los pequeños o que no beneficie a ninguno), pero no lo harán más proporcional en el sentido que le estamos dando a tal expresión.

Tal estrategia, podría alegarse, no soluciona el problema: después de todo, si tan sólo recogemos la incidencia de $\mathrm{M}$, estamos haciendo tabla rasa de todas las diferencias entre las fórmulas. A nuestro juicio, sin embargo: a) lo interesante es sobre todo la reflexión conceptual con respecto a la cuestión de los umbrales y la comprensión del concepto de proporcionalidad, y b) el cálculo que vamos a ofertar para la finura es superior a la alternativa actual, el Umbral Efectivo de Lijphart. Nos ocuparemos de esto último a continuación.

\section{EL CÁLCULO DE LA FINURA: DE HARE A DROOP}

A pesar de que venimos utilizando la cuota 1/M (la Cuota Natural) como indicador de la finura de cualquier sistema RP, es preferible adoptar en su lugar la cuota 100/M+1 (la cuota Droop). Ciertamente, la opción más obvia parece desde luego la operación 100/M: de modo espontáneo, lo que estableceríamos es que un sistema con 10 escaños funciona con una finura de un $10 \%$. Pero, como es sabido, Droop es sin duda un cálculo más adecuado.

Por un lado, siempre que un partido alcance Droop, logrará escaño con independencia de la fórmula ${ }^{10}$. Por otro, no puede existir el caso de que haya más partidos que $\mathrm{M}$ que consigan Droop. Esta segunda característica es especialmente relevante desde la perspectiva de la finura, que es la de la proporcionalidad Global. Desde la misma, todos los porcentajes marcados por los umbrales, excepto Droop, no resultan lógicos interpretados como índice de proporcionalismo de los sistemas. El Umbral de Precio Medio con $\mathrm{M}=10$, por ejemplo, señala un 5\%. Por tanto, si 10 partidos consiguen un 5\% de los votos, cada uno obtiene un

\footnotetext{
10 Por ello, la cuota Droop resulta compatible, como no podía ser de otra manera, con todos los valores recogidos en la tabla de los Umbrales del apartado 3.2: todos son inferiores a la misma, pero ninguno es superior.
} 
escaño y se llevan los 10 que hay en juego. Ahora bien, tales partidos sumarían el $50 \%$ de los votos: ¿dónde está entonces el 50\% de votos restante? Como indicadores del proporcionalismo del sistema, todas las medidas inferiores a Droop resultan incoherentes. El Umbral Efectivo de Lijphart, en especial, y a pesar del éxito que ha cosechado desde su aparición (Gallagher y Mitchell, 2005), es en este sentido especialmente desafortunado. Lijphart bebe de dos tradiciones y, creemos, ambas se encuentran desencaminadas.

La primera, teórico-matemática, es la de los umbrales, de la que ya nos hemos ocupado. Desde esta tradición, lo que Lijphart intenta es atrapar la influencia de la fórmula. Al final, sin embargo, no lo consigue: su Umbral ignora la fórmula en su cálculo y es universal, invariable sea cual sea la fórmula (como lo es Droop, y en ese sentido ambos se hallarían empatados, por así decir). Sin embargo, y al contrario que Droop (cuya justificación venimos intentando defender), su Umbral sale malherido de ese intento; está sujeto a la misma crítica que acabamos de ejemplificar para todos los umbrales inferiores a Droop: carece de lógica como indicador de la proporcionalidad del sistema ${ }^{11}$.

La segunda tradición, que podríamos denominar empírica, se encuentra en la estela del Umbral que sugieren Taagepera y Shugart en 1989 como una medida empírica y nacional (proponen la siguiente ecuación: 50\%/M). Lo que Lijphart persigue es así ofrecer un cálculo que resulte razonable para ser aplicado a (todas) las diferentes realidades empíricas. Aquí se han de asumir ciertos presupuestos (sobre el número de partidos, la fórmula empleada, el número de distritos, etc.) para que el Umbral «funcione» bien, presupuestos que Lijphart tiene que compartir en mayor o menor medida, ya que aplica su medida a los agregados nacionales. Taagepera ha continuado ofreciendo distintas medidas en la línea de dicho objetivo (Taagepera, 1989, 1998 y 2002). Con independencia del juicio que merezca tal enfoque empiricista (cfr. la crítica de Lin, que lo denomina Physics-style approach; Lin, 2003: 51), el mismo obedece a unos planteamientos y unos objetivos por completo diferentes a los de la presente Nota, cuyas pretensiones son más bien teóricas y conceptuales ${ }^{12}$.

11 Por ejemplo: con $\mathrm{M}=10$ el Umbral Efectivo es de un 6,8\%. Por tanto, si 10 partidos consiguen un $7 \%$ de los votos, cada uno obtiene un escaño y se llevan los 10 que hay en juego. Tales partidos sumarían el $70 \%$ de los votos: ¿qué ocurre entonces con el $30 \%$ de votos restante? Como sabemos, el origen de esta confusión es que, en su caso, el Umbral podría tener sentido para un determinado partido, pero no para el sistema en sí. Podría alegarse que Lijphart no construye su Umbral como un índice de proporcionalismo, sino tan sólo como un umbral de inclusión, sin referencia al ideal de la proporcionalidad. Pero el caso es que, en cuanto que viene señalado en porcentajes, su Umbral no puede escapar del imperio de la idea regulativa de proporcionalidad (Global). Es sencillamente incoherente un Umbral de Inclusión del sistema que señale un $7 \%$ con M=10: si el sistema impone un $7 \%$, entonces «caben» hasta 14 partidos. Se trata, bien mirado, del mismo tipo de incoherencia de los resultados electorales de ciertas dictaduras en las que ocurre que el nivel de participación (y, por descontado, de apoyo al líder) alcanza el $103 \%$, o del de esas encuestas en las que la suma de los porcentajes de los entrevistados arroja un $120 \%$.

12 Probablemente, esta aspiración (buscar un umbral universal), junto a la anterior (introducir la incidencia de la fórmula), explican que — por exceso de ambición, por así decir- el Umbral Efectivo de Lijphart haya sido criticado desde su aparición (Penadés, 1997) hasta nuestros días (Ruiz-Rufino, 2005b). A pesar de ello, a pesar de que ciertamente es complicado encontrar un fragmento más confuso, enmarañado y metodológicamente endeble que el que configuran las páginas en las que lo pre- 
En una Nota de Investigación anterior (Urdánoz, 2006b) sosteníamos que no es posible ofrecer una medida «intermedia» —o, en la expresión de Gallagher, un «feliz término medio»- entre los índices Absoluto y Relativo (índices que miden la proporcionalidad de los resultados). Tal intento conduce al índice de Mínimos Cuadrados, a nuestro juicio inconsistente. De modo similar, aquí sostenemos que tampoco es posible, a la hora de elaborar un índice de proporcionalidad de los sistemas, ofrecer a través de los umbrales un índice que recoja la incidencia de $\mathrm{M}$ y también de la fórmula (lo que sería otra suerte de «feliz término medio»), intento que desemboca, también inconsistentemente a nuestro juicio, en el Umbral Efectivo de Lijphart.

Por lo demás, aunque no estemos de acuerdo con la solución de Lijphart, vamos a continuación a apropiarnos de su propia obra (al modo, todo hay que decirlo, del peor vampirismo) para demostrar que nuestro Umbral «funciona» tan bien, cuanto menos, como el suyo. Podemos utilizar la metodología y los resultados de su celebrado estudio (Lijphart, 1994) y trasladarlos a la idea de finura. La ecuación que Lijphart ofrece para su Umbral Efectivo $(75 \% / \mathrm{M}+1)$ arroja un resultado que corre en paralelo a Droop, si bien es siempre un poco más pequeño. Así, para diferentes sistemas electorales (en los que sólo varía M, pues se da por supuesta una fórmula proporcional), los Umbrales Efectivos y los diferentes grados de proporcionalismo o finura (señalados por Droop) serían:

\begin{tabular}{|c|c|c|c|c|c|c|c|c|c|c|c|c|c|c|}
\hline & \multicolumn{14}{|c|}{ M } \\
\hline & 2 & 3 & 4 & 5 & 6 & 7 & 8 & 9 & 10 & 20 & 50 & 100 & 200 & 1000 \\
\hline UMBRAL EFECTIVO & 25,00 & 18,75 & 15,00 & 12,50 & 10,71 & 9,38 & 8,33 & 7.5 & 6,82 & 3,57 & 1,47 & 0,74 & 0,37 & 0,07 \\
\hline FINURA & 33,33 & 25,00 & 20,00 & 16,67 & 14,29 & 12,50 & 11,11 & 10,00 & 9,09 & 4,76 & 1,96 & 0,99 & 0,50 & 0,10 \\
\hline
\end{tabular}

Aunque Lijphart no interprete explícitamente su Umbral Efectivo como un «índice» de proporcionalismo de los sistemas, implícitamente lo concibe así, puesto que la hipótesis que trata de contrastar es: «a menor umbral (de los sistemas), mayor proporcionalidad (de los resultados)». En esa línea presenta la siguiente tabla, en la que divide los umbrales efectivos en cinco grandes grupos y los relaciona con la desproporcionalidad de los resultados ${ }^{13}$ (Lijphart, 1994: 98):

senta y justifica (Lijphart, 1994: 25-30), y a pesar de que, precisamente en respuesta a las críticas de Penadés, lo modificó de manera igualmente caprichosa e inconsistente (Lijphart, 1997), tal Umbral sigue siendo «la medida más frecuentemente usada en la literatura para caracterizar sistemas electorales» (Ruiz-Rufino, 2005b: 95).

13 La desproporcionalidad de los resultados se ofrece medida por el índice de los Mínimos Cuadrados (MC), medición que, a nuestro juicio, carece de excesivo fundamento (véase nuestra crítica en Urdánoz, 2006b), pero que es la que Lijphart defiende y la que se encuentra más extendida en la literatura; y por el índice de Loosemore-Hanby, que nosotros denominamos «Índice Absoluto» (y que es el que consideramos idóneo). 
Umbral Efectivo

Grupo A - 35\%

Grupo B - 12,9 a $18,8 \%$

Grupo C - 8 a $11,7 \%$

Grupo D - 4 a 5,9\%

Grupo E - 0,1 a 3,3\%
Ordenamientos con ese Umbral
Desproporcionalidad media de tales ordenamientos

$\mathrm{MC}(\%)$

Índice Absoluto (\%)

12

9

$\begin{array}{rr}12,44 & 16,71 \\ 7,24 & 9,54 \\ 5,74 & 9,01 \\ 3,68 & 6,47 \\ 2,29 & 4,35\end{array}$

Lijphart concluye que «la desproporcionalidad disminuye, de acuerdo con lo establecido en la hipótesis, conforme disminuye el Umbral Efectivo, y lo hace de modo monotónico y con diferencias bastante grandes de una a otra de las cinco categorías» (Lijphart, 1994: 100). En la medida en que se considere que tal conclusión demuestra, corrobora o apoya empíricamente que existe una relación entre el Umbral Efectivo y la desproporcionalidad, entonces también ha de establecerse lo mismo si, en vez de considerar el Umbral Efectivo, se considera la finura de los sistemas medida conforme a Droop, en cuyo caso obtendríamos la siguiente tabla:

\begin{tabular}{|c|c|c|c|}
\hline \multirow[b]{2}{*}{ Proporcionalismo } & \multirow{2}{*}{$\begin{array}{r}\text { Ordenamientos } \\
\text { con ese proporcionalismo }\end{array}$} & \multicolumn{2}{|c|}{ Desproporcionalidad media de tales ordenamientos } \\
\hline & & $\mathrm{MC}(\%)$ & Índice Absoluto (\%) \\
\hline Grupo A - 50\% & 12 & 12,40 & 16,72 \\
\hline Grupo B - 12,9 a $21,1 \%$ & 15 & 6,72 & 10,01 \\
\hline Grupo C - 10,2 a $11,7 \%$ & 7 & 4,29 & 7,01 \\
\hline Grupo D - 4 a $7,6 \%$ & 13 & 3,38 & 6,07 \\
\hline Grupo E - 0,2 a 3,8\% & 22 & 2,81 & 5,07 \\
\hline
\end{tabular}

Esta tabla no es más que la versión reducida de la siguiente (elaborada a partir de los datos del propio Lijphart ${ }^{14}$ ):

\footnotetext{
14 De la misma manera que Lijphart calcula su Umbral Efectivo teniendo en cuenta, cuando existen distritos, la media de escaños por país (por ejemplo, España sería un sistema electoral con 6,7 escaños, el resultado de dividir los 350 escaños que se eligen entre los 52 distritos del país), nosotros hemos hecho lo propio para calcular la finura mediante Droop. Los datos de Lijphart corresponden a su obra de 1994 (por lo que utilizan el cálculo para el Umbral Efectivo anterior a la modificación que el propio Lijphart introdujo en 1997), pero el fundamento de nuestra crítica es, creemos, idéntico con independencia de que se utilice uno u otro cálculo para el Umbral Efectivo.
} 


\begin{tabular}{|c|c|c|c|c|c|}
\hline \multirow[b]{2}{*}{ Sistema } & \multirow[b]{2}{*}{ Finura (\%) } & \multicolumn{2}{|c|}{ Desproporcionalidad (\%) } & \multicolumn{2}{|c|}{ Desproporcionalidad (\%) } \\
\hline & & $\mathrm{MC}$ & Índice Absoluto & $\mathrm{MC}$ & Índice Absoluto \\
\hline CAN & 50,00 & 11,3 & 13,8 & & \\
\hline EE.UU. & 50,00 & 5,4 & 5,9 & & \\
\hline $\mathrm{RU} 1$ & 50,00 & 10,5 & 12,9 & & \\
\hline IND2 & 50,00 & 16,7 & 23,8 & & \\
\hline $\mathrm{NZ}$ & 50,00 & 10,6 & 12,4 & & \\
\hline RU2E & 50,00 & 19,45 & 23,9 & 12,40 & 16,72 \\
\hline FRA3 & 50,00 & 14,1 & 20,8 & & \\
\hline FRA6 & 50,00 & 11,8 & 18,6 & & \\
\hline AUL1 & 50,00 & 9,6 & 13,2 & & \\
\hline AUL2 & 50,00 & 8,5 & 12,2 & & \\
\hline AUL3 & 50,00 & 10,2 & 13,9 & & \\
\hline IND1 & 45,25 & 20,7 & 29,2 & & \\
\hline IRL1 & 21,05 & 3,4 & 5,2 & & \\
\hline IRL2E & 21,05 & 10,8 & 16,3 & & \\
\hline JPN2 & 20,20 & 5,7 & 8,7 & & \\
\hline MAL1 & 16,67 & 3,7 & 5,1 & & \\
\hline MAL2 & 16,39 & 3,7 & 4,4 & & \\
\hline GRE5 & 16,29 & 4 & 5,8 & & \\
\hline FRA1 & 16,16 & 3,6 & 5,4 & & \\
\hline GRE3E & 15,87 & 2,7 & 5,7 & & \\
\hline GRE2 & 15,87 & 10,9 & 16,2 & 6,72 & 10,01 \\
\hline FRA5 & 14,73 & 7,2 & 12,7 & & \\
\hline LUX2E & 14,29 & 13,1 & 20,2 & & \\
\hline CR1 & 13,46 & 3,8 & 5,4 & & \\
\hline AUT1 & 13,16 & 3,6 & 5 & & \\
\hline GRE1 & 13,04 & 15,7 & 18,9 & & \\
\hline ESP1 & 12,94 & 8,9 & 15,1 & & \\
\hline NOR1 & 11,76 & 8,5 & 10,7 & & \\
\hline NOR2 & 11,36 & 4,3 & 7 & & \\
\hline CR2 & 10,94 & 4,1 & 7,2 & & \\
\hline SU1 & 10,87 & 2,3 & 5 & 4,29 & 7,01 \\
\hline SUE2 & 10,86 & 3,5 & 5,2 & & \\
\hline SUE2 & 10,79 & 2,3 & 3,4 & & \\
\hline JPN1 & 10,26 & 5 & 10,6 & & \\
\hline BEL2E & 7,69 & 6,1 & 11,35 & & \\
\hline POR1 & 7,46 & 4,2 & 6,8 & & \\
\hline FIN & 7,04 & 2,8 & 5 & & \\
\hline LUX1 & 6,66 & 3,1 & 4,6 & & \\
\hline DIN4E & 6,12 & 7,4 & 14 & & \\
\hline ITA4E & 5,81 & 1,1 & 2,2 & & \\
\hline ITA1 & 5,28 & 1,5 & 2,8 & 3,38 & 6,07 \\
\hline ITA2 & 5,06 & 3,6 & 5,8 & & \\
\hline ITA3 & 4,87 & 2,7 & 4,6 & & \\
\hline AUT2 & 4,69 & 1,4 & 2,1 & & \\
\hline BEL1 & 4,08 & 3,2 & 5,9 & & \\
\hline POR2E & 4,00 & 4,1 & 8 & & \\
\hline GRE3E & 4,00 & 2,7 & 5,7 & & \\
\hline
\end{tabular}




\begin{tabular}{|c|c|c|c|c|c|}
\hline \multirow[b]{2}{*}{ Sistema } & \multirow[b]{2}{*}{ Finura $(\%)$} & \multicolumn{2}{|c|}{ Desproporcionalidad (\%) } & \multicolumn{2}{|c|}{ Desproporcionalidad (\%) } \\
\hline & & $\mathrm{MC}$ & Índice Absoluto & MC & Índice Absoluto \\
\hline HOL3E & 3,85 & 4,7 & 8,4 & & \\
\hline ALE1 & 2,66 & 3,8 & 8,4 & & \\
\hline ISL1 & 1,89 & 7,4 & 9,7 & & \\
\hline ALE2 & 1,81 & 3,6 & 7,2 & & \\
\hline ESP2E & 1,64 & 5,45 & 11,2 & & \\
\hline ISL2 & 1,63 & 2,8 & 3,9 & & \\
\hline MAL3 & 1,43 & 0,3 & 0,4 & & \\
\hline ALE4E & 1,27 & 4,2 & 6,6 & & \\
\hline ALE6E & 1,27 & 1,9 & 4,2 & 2,81 & 5,07 \\
\hline FRA4E & 1,22 & 6 & 11,7 & & \\
\hline HOL1 & 0,99 & 1,2 & 2,7 & & \\
\hline ISR1 & 0,83 & 2,4 & 5,7 & & \\
\hline ISR3 & 0,83 & 2,6 & 5,7 & & \\
\hline ISR2 & 0,83 & 0,8 & 2,3 & & \\
\hline DIN1 & 0,67 & 1,8 & 2,4 & & \\
\hline HOL2 & 0,66 & 1,3 & 2,9 & & \\
\hline NOR3 & 0,60 & 3,6 & 5 & & \\
\hline DIN3 & 0,57 & 1,7 & 3,3 & & \\
\hline DIN2 & 0,57 & 2 & 2,8 & & \\
\hline SUE3 & 0,29 & 1,6 & 2,5 & & \\
\hline ALE3 & 0,20 & 2,1 & 3,3 & & \\
\hline ALE5 & 0,20 & 0,6 & 1,3 & & \\
\hline
\end{tabular}

Medida por la sencilla cuota Droop, la variable de la finura puede así ostentar idénticos méritos como aspirante al título de «verificada empíricamente» que los que pueda lucir el Umbral Efectivo. Títulos que, por lo demás, aunque ésa es otra cuestión, no son, a nuestro juicio, en absoluto afortunados ${ }^{15}$.

\section{LA FORMULACIÓN DE LA RELACIÓN, EL SESGOY EL AZAR}

La idea de que un sistema es más proporcionalista cuanto más fino (cuanto menor sea Droop) es intuitivamente obvia y fue señalada muy tempranamente (Humphreys, 1911). El primero en demostrarlo empíricamente fue Rae (1967) y, tras él, la tesis ha sido apuntalada por la Ciencia Política. De hecho, lo que aquí hemos venido llamando «finura» se denomina habitualmente «Magnitud de Distrito» y se considera el factor decisivo no sólo en relación con la proporcionalidad, sino con respecto a otras muchas variables (Taagepera y Shugart,

15 Este tipo de «comprobaciones teóricas» aplicadas a los agregados nacionales adolecen, creemos, de demasiados problemas. En primer lugar, los datos empíricos no pueden aportar demasiado a lo que establezca una teoría de índole matemática (y enseguida veremos hasta qué punto el azar tiene una influencia considerable aquí). En segundo lugar, y sobre todo, no estamos de acuerdo en los datos que el propio Lijphart, y en general toda la Ciencia Política, asumen como «empíricos» o «científicos»: existen lagunas en el propio proceso de recolección de los mismos (algo de esto apuntábamos en la nota 27 de Urdánoz, 2006b). A pesar de la importancia de ambas cuestiones, no podemos adentrarnos aquí en ellas. 
1989; Gallagher, 1991; Lijphart, 1994; Cox, 1997; Benoit, 2001). Con todo, la relación entre $\mathrm{M}$ y proporcionalidad no acaba de asumirse con rotundidad debido básicamente a dos factores que intervienen en la misma de una manera un tanto confusa y, a nuestro juicio, todavía no suficientemente elucidada en el ámbito teórico: los resultados y la fórmula.

\subsection{Proporcionalidad y resultados}

La relación entre mayor finura y mayor proporcionalidad de los resultados se suele presentar como algo evidente pero, a la vez, «no lineal». Desde los orígenes de la investigación (Rae: «The positive relationship between proportionality and district magnitude is curvilinear: as district magnitudes are increased, proportionality increases at a decreasing rate», en Rae, 1971: 181, cursiva nuestra) hasta sus últimos avances (Penadés: «el incremento de la magnitud correlaciona con una tendencia de los sistemas RP a aproximarse a la proporcionalidad perfecta, pero esta tendencia no es un orden", en Penadés, 2000: 320, ídem), se sabe que, por un lado, es cierto que a más finura, más proporcionales son los resultados, pero que, por otro, la relación no es monótona o continua, sino que está plagada de altibajos (Penadés, 2000: 313). ¿Puede, entonces, afirmarse con rotundidad que a mayor finura, mayor proporcionalismo del sistema? Intentaremos desenredar esta cuestión.

A nuestro juicio, la razón de la «no linealidad», de los altibajos, descansa en el hecho de que existe con respecto a la proporcionalidad de los resultados un factor de arbitrariedad, de azar, por completo renuente a ser asimilado mediante cualquier hipótesis causal (como todo factor genuinamente arbitrario y azaroso, por otra parte). Un sistema con 3 escaños es más proporcionalista (finura del 25\%) que otro con 2 (finura del $33 \%$ ). Sin embargo, si los resultados electorales resultan ser, habiendo dos partidos, uno el $51 \%$ de los votos y el otro el $49 \%$ de los mismos, entonces es fácil ver que obtendremos resultados más proporcionales con el sistema menos proporcionalista. Esta contingencia, este capricho en la forma que adopta la distribución del voto, podría ocurrir en cualquier realidad política y para cualquier cantidad posible de M. Por poner un caso extremo, un sistema con $M=1$ (finura del $50 \%$ ) podría arrojar resultados completamente proporcionales (si se logra la unanimidad) y otro con $\mathrm{M}=100$ (finura del $1 \%{ }^{16}$ ) podría arrojar resultados muy desproporcionales (imaginemos 100 partidos con 2 votos cada uno y otros 800 con 1 voto por cabeza: la desproporcionalidad sería del $80 \%)$.

Tal dependencia del azar, sin embargo, no deja de inscribirse en una pauta clara. Los altibajos existen, pero dentro de un orden: ya Rae demostró que el fuerte cambio en la tendencia de la curva de proporcionalidad se produce precisamente hacia la magnitud de 20.

$160,99 \%$, más exactamente $(=100 / 101)$. 
En otras palabras: importa mucho, muchísimo, si en un sistema pasamos de 1 a 10 escaños. Importa poco si pasamos de magnitud 10 a 20. Importa casi nada si pasamos de 20 a 60. Importa asintóticamente nada, por decirlo de algún modo, si pasamos de 60 a 150 o más. Cuando decimos «importa» queremos decir, obviamente, «afecta a la desproporcionalidad» de los resultados ( $\mathrm{y}$ a la fragmentación, pero de tal cuestión no nos estamos ocupando aquí).

Esta pauta no es casual. Se produce debido a que, dentro de un margen, los posibles universos electorales reales no son infinitos y se circunscriben a un conjunto relativamente acotado de posibilidades. Es decir: aunque sean matemáticamente posibles, no existen resultados electorales con miles de partidos empatados en apoyo electoral, por ejemplo. Los resultados pueden ser caprichosos, pero en general se engloban en posibilidades circunscritas a una horquilla de, pongamos, 2 a 8 partidos con posibilidades de representación, por lo que el factor azar queda bastante restringido ${ }^{17}$.

Más allá de eso, sin embargo, importa insistir en que la posibilidad de existencia de tales contingencias empíricas dependientes del azar no debería menoscabar la fortaleza teórica de la afirmación de que el proporcionalismo de un sistema electoral (entendido como capacidad de tal sistema para arrojar resultados proporcionales) aumenta con la finura (entendida como mayor número de escaños o como menor moneda de cambio mediante la que repartir la entidad «representación»). En consecuencia, puede establecerse sin ambages que un sistema es más proporcionalista cuanto más fino es o, en otras palabras, cuantos más escaños oferte.

Se puede observar ahora la justificación de la terminología introducida: los sistemas pueden ser más o menos proporcionalistas, no más o menos proporcionales. Un mayor proporcionalismo no garantiza necesariamente unos resultados más proporcionales: se trata de una relación de probabilidad, no de necesidad, lo que implica que el azar tiene siempre una influencia. La inclusión del sufijo «ismo» intenta atrapar esa inevitable sujeción a la probabilidad de todo sistema electoral.

Un sistema no proporcionalista sino proporcional («puramente proporcional», como suele decirse) sería una posibilidad que podría tan sólo diseñarse a posteriori, es decir: para cualesquiera resultados electorales, podría siempre configurarse ad hoc un determinado

\footnotetext{
17 Sobra decir que los comentarios vertidos en estos párrafos son meramente tentativos y que tan sólo pretenden dibujar, de una manera muy rudimentaria y elemental, el núcleo central de la explicación que parece aquí pertinente. En la medida en que ésta sea cierta, entonces los resultados generados por ordenador (donde todas las posibilidades, también las caprichosas, tienen cabida) deberían arrojar una relación más débil entre proporcionalismo y desproporcionalidad que los extraídos de la realidad política (en la que no existe tanto margen para el azar), pero repetimos que todo esto es muy aproximativo.
} 
sistema electoral que les hiciera justicia y no arrojara ninguna desproporcionalidad en el reparto. Pero, a priori, diseñados con anterioridad a la votación, como necesariamente han de ser todos los sistemas electorales del universo empírico parlamentario, los sistemas electorales sólo pueden ser (más o menos) proporcionalistas. Bajo esta perspectiva terminológica y conceptual, por tanto, ni existen ni pueden existir «sistemas proporcionales» en nuestras democracias representativas.

\subsection{Fórmula y proporcionalidad}

La intrincada incidencia de la fórmula ha influido, sin duda, en el hecho de que los criterios para establecer cuándo un sistema es más proporcionalista que otro no se hayan establecido con nitidez por parte de la Ciencia Política. Como venimos defendiendo, por un lado el uso de los umbrales es muy equívoco en este terreno. Por otro, comprobar «empíricamente» cualquier tipo de hipótesis es complicado cuando el azar tiene una influencia tan notable como la que acabamos de ver (si algo tan a priori evidente como que un sistema con $M=1$ es «menos proporcional» que otro con $M=100$ puede verse refutado en ciertos casos empíricos por unos concretos resultados electorales, intentar calibrar la diferencia entre dos sistemas con $\mathrm{M}=10$ pero uno con Precio Mínimo y otro con Precio Medio, por ejemplo, puede resultar sencillamente quimérico).

Con todo, a nuestro juicio, el elemento que más confusión ha generado han sido las diversas «escalas de proporcionalidad» elaboradas para las fórmulas. Con independencia de la mayor o menos consistencia de las mismas (cuestión por lo demás crucial pero en la que no podemos entrar aquí), el problema de estas escalas de proporcionalidad de las fórmulas es que, si no se elucida con nitidez su relación con $\mathrm{M}$, pueden interpretarse sin mayores cautelas como escalas de proporcionalidad de los sistemas. Aunque existía considerable evidencia de que la finura influye en la proporcionalidad en mayor grado que la fórmula (Taagepera y Laakso, 1980: 443; Taagepera y Shugart, 1989: 112-125; Gallagher, 1992: 43), de nuevo la aportación de Lijphart ha podido ser aquí contraproducente. En su estudio de 1994, Lijphart afirma haber encontrado una relación entre la fórmula y el grado de desproporcionalidad. Utiliza la siguiente escala de desproporcionalidad de las fórmulas:

1) Mayoría relativa.

2) Otras fórmulas mayoritarias (mayoría absoluta y voto alternativo).

3) Las fórmulas RP menos proporcionales (D’Hondt y RM-Imperiali). 
4) Las intermedias (RM-Droop, VUT, Ste. Laguë Modificada y VUNT).

5) La fórmula más proporcional (RM-Hare).

Mediante tal escala, expone la pauta empírica en la siguiente tabla:

\begin{tabular}{lrr} 
Fórmula & $\begin{array}{r}\text { Número de países } \\
\text { que la utilizan }\end{array}$ & $\begin{array}{r}\text { Desproporcionalidad media } \\
\text { (Índice Absoluto) (\%) }\end{array}$ \\
\hline Mayoría relativa & 7 & 17,8 \\
Otras mayoritarias & 5 & 14,4 \\
Menos proporcionales & 32 & 8,39 \\
Medias & 13 & 6,29 \\
Proporcional & 12 & 3,2
\end{tabular}

A partir de la misma, afirma: «De acuerdo con lo señalado por la hipótesis, el porcentaje de desproporcionalidad disminuye desde la fórmula de mayoría relativa a la RM-Hare. Esta disminución es a la vez monotónica y espectacular» (Lijphart, 1994: 100). El problema aquí no es la pauta empírica, que obviamente existe; el problema es teórico: lo inverosímil es la propia hipótesis que Lijphart somete a prueba, el hecho de que la considere digna de ser tenida en cuenta. A partir de un conocimiento sólido de la proporcionalidad no es posible esperar que, sin controlar $\mathrm{M}$, pueda hallarse una pauta como la que Lijphart propone (y además encuentra, circunstancia que ha de achacarse al azar). La hipótesis carece de fundamento: no podemos prever el proporcionalismo de un sistema electoral única y exclusivamente gracias a la fórmula, pues ésta sólo puede maniobrar dentro de los márgenes de la finura que imponga $\mathrm{M}$. De hecho, si revelamos el valor medio de la finura para los grupos que Lijphart utiliza, obtenemos lo siguiente:

\begin{tabular}{lrrr} 
Fórmula & $\begin{array}{r}\text { Número de sistemas } \\
\text { que la utilizan }\end{array}$ & $\begin{array}{r}\text { Finura de } \\
\text { tales sistemas (\%) }\end{array}$ & $\begin{array}{r}\text { Desproporcionalidad } \\
\text { media } \\
\text { (Índice Absoluto) (\%) }\end{array}$ \\
\hline Mayoría relativa & 7 & 50 & 17,8 \\
Otras mayoritarias & 5 & 50 & 14,4 \\
Menos proporcionales & 32 & 1,90 & 8,39 \\
Medias & 13 & 1,86 & 6,29 \\
Proporcional & 12 & 0,88 & 3,2
\end{tabular}

Por tanto, aunque empíricamente cualquiera de los dos factores (la fórmula o la finura) podría postularse como causa que incide en la proporcionalidad, a nuestro juicio al me- 
nos tan sólo a la finura le cabe reivindicarse teóricamente como mecanismo explicativo $^{18}$

Muy al contrario, sin embargo, la lectura de Lijphart produce la ilusión de que la influencia de la fórmula en el proporcionalismo del sistema (así, en abstracto y sin relación con M) no sólo es teóricamente consistente, sino que además se halla empíricamente contrastada. De ahí que la cuestión de la «proporcionalidad de las fórmulas», al presentarse con la irresistible aureola que otorga el título de «verificada empíricamente», haya entorpecido notablemente la comprensión del proporcionalismo de los sistemas. No pocos autores (dos ejemplos, entre otros muchos, serían Carter, 2005: 156-160, y Ruiz-Rufino, 2005b: 100 y 104) parecen interpretar que la escala de proporcionalidad de las fórmulas deviene automáticamente escala de proporcionalidad de los sistemas, y, en consecuencia, estiman que los resultados electorales deberían ser más proporcionales en aquellos sistemas que utilizan una fórmula «más proporcional». Como venimos intentando demostrar, tal planteamiento carece de fundamento si no se tiene en cuenta la finura de cada sistema.

\subsection{Finura y proporcionalismo}

A nuestro juicio, y más allá de mayores o menores evidencias empíricas, hay buenas razones teóricas para establecer lisa y llanamente que la finura dibuja por sí misma una escala de proporcionalismo de los sistemas, de manera tal que — con independencia de la fórmula, supuesto que ésta sea proporcional - mediante la misma pueden ordenarse todos ellos. Así, un sistema con $\mathrm{M}=10$ es más proporcionalista que otro con $\mathrm{M}=9$. La fórmula nos informará sobre el sesgo, pero difícilmente hará «más proporcional» al sistema menos fino: su incidencia no revierte en términos de mayor o menor proporcionalidad, sino en los de mayor o menor beneficio a los pequeños o a los grandes (o, lo que es lo mismo, mayor o menor sesgo, un sesgo que sólo podrá tomar forma y actuar, en su caso, dentro de los márgenes del proporcionalismo del sistema ${ }^{19}$ ).

18 Si un estudio del conjunto de niños de cualquier país arrojara una relación empírica entre «mayor largura de brazos» y «mayor inteligencia», nadie relacionaría causalmente un hecho con otro, pues la teoría previa no permitiría tal ilación. Así, se achacaría la pauta al azar o se buscaría otra explicación, otra circunstancia empírica que, además de la largura de los brazos y coincidiendo con ella, pudiera explicar el hecho. Encontraríamos enseguida que no es dicha largura lo que se relaciona causalmente con la inteligencia, sino más bien la mayor edad (que implica normalmente brazos más largos). Lijphart incurre, por tanto, en una de las así llamadas falacias Non Causa pro Causa, o Falsa Causa (Fischer, 1970: cap. VI), y la explicación radi$\mathrm{ca}$, a nuestro juicio, en que el confuso conocimiento teórico del que parte le permite identificar sin mayores problemas como «causa» algo que ni lo es ni puede serlo. Hubiera sido más coherente achacar la relación empírica al azar, pero la propia configuración conceptual en la que está instalado - a nuestro juicio equívoca - le empuja a enfocar la cuestión de modo causal.

19 Esto, ciertamente, deja sin solucionar la incidencia de la fórmula dentro de cada valor de M. Si M=10, ¿es «más proporcional» un sistema con Precio Mínimo u otro con Precio Medio, con independencia de que ya sepamos que uno está más sesga- 
Quizás pueda aquí traerse a colación el argumento rawlsiano del «velo de la ignorancia». Supongamos que tengamos que establecer, a priori, los rasgos fundamentales de un sistema de reparto guiado por el ideal de la proporcionalidad mediante el cual, a posteriori (es decir: una vez que desaparezca el velo y averigüemos si somos grandes o pequeños), se va a proceder a distribuir un bien cualquiera. Establecida una fórmula proporcional, en el interés de cualquier sujeto racional estará aumentar la finura siempre y en todo caso, pues es obvio que eso supone automáticamente mayores posibilidades de recibir una porción del bien más proporcional: si la unidad de medida es pequeña, si el sistema es fino, tanto los grandes como los pequeños podrán recibir una porción más ajustada a lo que les corresponde.

De hecho, esta intuición explica también, de nuevo, ciertas confusiones comunes con respecto a la interpretación habitual de los umbrales. Supongamos dos sistemas RP idénticos en todo excepto en la fórmula. Si se nos ofrecen los umbrales para ambos y resultan ser, por ejemplo, 10 y 15\%, espontáneamente tenderemos a pensar que el primer sistema es más proporcional. ¿Por qué? Es fácil ver aquí trabajando la idea de finura: consideramos que cuanto más pequeña (más fina) sea la «unidad de medida», más proporcional será el sistema. Pero no porque tengamos alguna clase de predilección o parcialidad por los partidos pequeños (que es lo que ocurre con los umbrales, elaborados inevitablemente desde un partido concreto: el pequeño), sino al contrario: porque intuimos que, si el sistema es capaz de representar al partido pequeño, eso significa que es muy fino y damos por hecho que, en consecuencia, también al grande lo representará con justicia (entendida aquí la justicia, obviamente, como proporcionalidad).

\section{CONCLUSIONES Y PROPUESTAS TERMINOLÓGICAS}

Lo que esta Nota de Investigación persigue no es tanto descubrir algo (y menos todavía un algo tan obvio como que la proporcionalidad depende de $\mathrm{M}$ ) como más bien desenredar algunos aspectos conceptuales que, a nuestro juicio, se abordan de forma especialmente confusa $^{20}$. En la medida que se compartan las precisiones conceptuales aquí introducidas,

do que el otro? Además, puede plantearse la posibilidad de que la fórmula influya más con ciertos valores de M que con otros. Para dar una respuesta a tales cuestiones habría que afrontar la cuestión de la proporcionalidad de las fórmulas (¿qué fórmula es más proporcional?), cuestión que ha de quedar pendiente de un ulterior tratamiento.

20 Queremos remarcar lo establecido en la introducción: nuestros «sistemas electorales» no son los «sistemas electorales» pretendidamente empíricos habituales en Ciencia Política (como, por ejemplo, «el sistema electoral español», una construcción terminológica a nuestro juicio confusa). Nuestros sistemas electorales son de laboratorio. A veces ocurre que también son de verdad, es decir: existen en la realidad empírica, como por ejemplo los sistemas electorales de Israel y Holanda o los de las Comunidades Autónomas de Madrid o Navarra, en los que sólo encontramos una fórmula y un valor para M (y, en su 
quizás sea de utilidad proponer ciertas convenciones terminológicas como conclusiones de la misma.

En primer lugar, algunas que ya hemos introducido y cuya oportunidad quizás se vislumbre mejor ahora:

- Los sistemas no son más o menos proporcionales, sino más o menos proporcionalistas. El proporcionalismo es, sencillamente, la mayor o menor capacidad de un determinado sistema para producir resultados proporcionales y depende por completo, dando por descontada una fórmula proporcional, del número de escaños (finura). Un sistema que reparte escaños con una finura del $3 \%$ es más proporcionalista que uno que lo hace con una del $5 \%$.

- No es posible introducir la incidencia de la fórmula en términos de proporcionalidad, pues ésta no afecta al proporcionalismo, sino al sesgo. Por ello, las fórmulas proporcionales no son «más o menos proporcionales», sino más o menos sesgadas (hacia los grandes o hacia los pequeños). La Teoría de los Precios (Urdánoz, 2007) ofrece al respecto una nomenclatura iluminativa y sistemática.

- Únicamente los resultados pueden ser más o menos desproporcionales (aquí puede admitirse, por una cuestión de economía lingüística, la expresión resultados «más o menos proporcionales», pero sólo como el equivalente semántico de «más o menos desproporcionales»).

En segundo lugar, otras convenciones relativas al uso de los términos «Barrera», «Finura» y «Umbral». Las dos primeras son variables del sistema electoral y dependen inevitablemente de la concepción Global de la proporcionalidad:

- La barrera es un porcentaje de votos legalmente exigido para empezar a recibir escaños. Es una decisión ad hoc del legislador y puede existir o no.

- La finura señala el porcentaje de votos mediante el que se reparten los escaños (por ejemplo, una finura del 3\% señala que un partido recibirá al menos un escaño por cada

caso, una barrera). Pero, más allá de eso, lo que nos interesa de momento es el terreno conceptual, y desde ese terreno aún no hemos dado el paso a realidades institucionales más complejas. Tales realidades incluyen normalmente elementos que dificultan el tránsito de lo teórico a lo empírico: división en distritos, diferencias en el valor de voto, dobles niveles, etc. Recientemente, Rubén Ruiz-Rufino (2005a: cap. 3) ha ideado un método para atrapar cuantitativamente la influencia del número y el tamaño de los distritos (sin duda, uno de los elementos empíricos más complejos de abordar teóricamente) que, inicialmente, resulta prometedor (aunque, a nuestro juicio, el número de partidos no debería incluirse en el cálculo, como hemos dicho). Con todo, aquí no podemos sino dejar constancia de tal aportación y posponer la indagación de sus posibles potencialidades. 
lote del $3 \%$ de los votos que consiga). Es una consecuencia directa de $\mathrm{M}$ y, por tanto, existe siempre y necesariamente para cualquier sistema.

Así, la barrera es un requisito a partir del cual empezará a funcionar la finura o proporcionalismo del sistema. Es importante utilizar los términos adecuadamente, y una imagen gráfica a veces es la mejor explicación: una barrera es algo que se salta o se supera una sola vez; la finura es algo con lo que se juega continuamente una vez que se ha superado la barrera. La barrera puede ser más baja que el grado de finura: en tal caso es papel mojado y no sirve para nada. Por ejemplo, establecer una barrera del $3 \%$ en un sistema proporcionalista al 25\% ( $M=3)$. Sería equivalente a un Casino en el que las fichas cuestan 25 euros cada una y en el que hay un letrero que avisa a los clientes de que está prohibido jugar a no ser que tengan un mínimo de 3 euros. Dicho letrero (la barrera) es redundante, carece de sentido, sobra: el mínimo que hay que tener es, obviamente, de 25 euros. Si, al contrario, la barrera es más alta que la finura, entonces pasa a ser un elemento contradictorio con el proporcionalismo del propio sistema. Por ejemplo, si en un sistema proporcionalista al $1 \%(M=99)$ existe una barrera del $5 \%$ para poder entrar en el reparto, ambos cálculos se contradicen. Aquí nuestro Casino vende las fichas a 1 euro, pero se prohíbe jugar a todo aquel que no posea un mínimo de 5 euros. En este caso la barrera impide jugar a clientes que podrían comprar fichas (alguien, por ejemplo, con 4 euros), lo cual es en buena medida incoherente 21 .

La expresión «umbral», por último, es ambigua y ha de adjetivarse inevitablemente. Si estamos hablando, como es habitual, de un umbral «del sistema» (entendido como el porcentaje de votos necesario para obtener el primer escaño), entonces:

- Sólo la proporcionalidad Global tiene sentido.

- Tal umbral «del sistema» coincidirá o bien con la finura o bien con la barrera (cuando ésta sea más elevada que la finura). Por tanto, tal variable no aporta nada diferente a ambas y, en consecuencia, parecería aconsejable abandonar el uso de la denominación «umbral» aplicada a un sistema.

21 Esta incoherencia es especialmente difícil. Penadés afirma que es un problema que «la literatura todavía no ha resuelto» (Penadés, 1997: 61). Un intento de delimitar los diferentes efectos de los umbrales y las barreras (que ya Penadés adelanta o insinúa: Penadés, 1997: 65-66) puede verse en Anckar (1997). A nuestro juicio, es probable que toda la aproximación de Anckar al problema se origine única y exclusivamente debido a que utiliza el índice de los Mínimos Cuadrados para medir la desproporcionalidad de los resultados. Con el Índice Absoluto, la propia hipótesis (Anckar, 1997: 512, tabla 4) probablemente ni se plantearía. En la medida en que sea así (remarcamos el probablemente), entonces puede deducirse hasta qué punto un tipo de medición de la desproporcionalidad u otro puede desviar la investigación. Por lo demás, la interrelación entre barrera y finura sólo puede ser abordada satisfactoriamente desde una perspectiva normativa relacionada con el ideal de la representación proporcional (y no desde una meramente lógico-matemática centrada en el concepto de proporcionalidad, que es desde la que pretende estar redactada la presente Nota). 
La expresión «umbral» quedaría así restringida a los umbrales «de partido»: un cálculo gracias al cual podemos estimar los votos necesarios para que un determinado partido adquiera un escaño (o los que sea). Los umbrales dependen del número de partidos y de la concepción de la proporcionalidad que imponga la fórmula: Global si es de cuota, Binaria si es de divisor. Un partido puede saber que necesita un $10 \%$ de los votos para lograr un escaño, pero eso no implica que el proporcionalismo del sistema sea entonces del $10 \%$. Como hemos intentado establecer, son cosas considerablemente distintas.

Estas distinciones intentan lograr no sólo una mayor precisión conceptual, sino también algo tan obvio como evitar ciertos equívocos a los que puede conducir utilizar una misma denominación («umbral») para designar entidades diferentes (umbrales, barrera, finura) ${ }^{22}$. Lo mismo sucede con el término «proporcionalidad», que se usa aplicado indistintamente a sistemas, fórmulas y resultados, lo que está en el origen de múltiples y venerables malentendidos. Aquí hemos distinguido en su interior aspectos diferentes: proporcionalismo (de los sistemas), sesgo (de las fórmulas) y desproporcionalidad (de los resultados).

Más allá de lo que se piense de la oportunidad de tales convenciones, creemos haber demostrado que, de cara a la medición del proporcionalismo de los sistemas RP, existen sólidas razones para desechar los umbrales y las variables relacionadas con tal filosofía —como el Umbral Efectivo- y utilizar la idea de finura ${ }^{23}$. Idea que, además, y no es poco, resulta ser también considerablemente intuitiva, sencilla e inteligible.

\section{BIBLIOGRAFÍA}

ANCKAR, C. (1997): «Determinants of Disproportionality and Wasted Votes», Electoral Studies, 4: 501-515.

BALINSKI, M. L., y YOUNG, H. P. (1982): Fair Representation: Meeting the Ideal of One Man, One Vote, Yale University Press, New Haven.

BENOIT, Kenneth (2000): «Which electoral formula is the most Proportional? A new look with new evidence», Political Analysis, 8, 4:381-387.

- (2001): «District magnitude, electoral formula, and the number of parties», European Journal of Political Research, 39 (2): 203-224.

22 Anckar, por ejemplo, parece confundir, bajo la denominación única de threshold, lo que aquí llamaríamos «umbrales» con «barreras» (Anckar, 1997: 506).

23 En la medida en que la justificación de las convenciones se comparta, entonces la habitual expresión «Umbral de Inclusión del sistema electoral» debería desecharse: un sistema no puede tener un Umbral, sino finura (y, en su caso, una barrera), y haríamos bien en introducir términos diferentes para entidades diferentes. A nuestro juicio, además, la cuota Droop - la finura- ostenta propiedades analíticas y conceptuales superiores también (de hecho: sobre todo) en el terreno de la mayoría (es decir: resulta perfectamente válida para los sistemas mayoritarios). Pero ésa es otra historia y debe ser contada en otra ocasión. 
CARTER, E. (2005): The Extreme Right in Western Europe: Success or Failure?, Manchester University Press, Manchester.

COLOMER, J. M. (ed.) (2004): Handbook of Electoral System Choice, Palgrave-Macmillan, London y New York. Trad. española: Cómo votamos. Los sistemas electorales del mundo: presente, pasado y futuro, Gedisa, Barcelona, 2004.

COX, Gary W. (1997): Making Votes Count: Strategic Coordination in the World's Electoral Systems, Cambridge University Press, Cambridge.

FISCHER, D. H. (1970): Historians' Fallacies: Toward a Logic of Historical Thought, Harper \& Row.

GALLAGHER, Michael (1991): «Proportionality, Disproportionality and Electoral Systems», Electoral Studies, 10: 33-51.

GALLAGHER, Michael (1992): «Comparing Proportional Representation Electoral Systems: Quotas, Thresolds, Paradoxes and Majorities», British Journal of Political Science, 22: 469-496.

GALLAGHER, Michael, y MITCHELL, Paul (2005): The politics of electoral systems, Oxford University Press, New York.

GROFMAN, Bernard (1975): «A review of macro elections systems», Sozial Wissenschaftliches Jahrbuch fur Politik, 4: 303-352.

GROFMAN, B. (2001): «A note of caution in interpreting the threshold of exclusion», Electoral Studies, 20 (2): $299-303$.

HUMPHREYS, John H. (1911): Proportional Representation, Methuen and Co., London.

LAAKSO, M. (1979): «Thresholds of Proportional Representation reanalyzed», Munich Social Science Review, 1: 19-28.

LAKEMAN, Enid (1970): How Democracies Vote: a Study of Electoral Systems, Faber \& Faber, Londres (3. ${ }^{\text {a }}$ ed. revisada).

LIJPHART, A. (1986): «Degrees of proportionality of proportional representation formulas», en B. Grofman y A. Lijphart (eds.), Electoral laws and their political consequences, Agathon Press, Nueva York, pp. 170-179.

- (1994): Electoral Systems and Party Systems. A Study of Twenty-Seven Democracies, 1945-1990, Oxford University Press, Oxford.

- (1997): «The Difficult Science of Electoral Science: A commentary on the critique by Alberto Penadés», Electoral Studies, 16, 1: 73-77.

LIJPHART, Arendt, y GIBBERD, Robert W. (1977): «Threesolds and Payoffs in List Systems of R.P.», European Journal of Political Research, 5: 219-230.

LIN, J.-W. (2003): «Looking for the magic number: the optimal district magnitude for political parties in d'Hondt PR and SNTV», Electoral Studies, 22: 49-63.

LOOSEMORE, Johh, y HANBY, Victor J. (1971): «The Theoretical Limits of Maximum Distorsion: Some Analityc Expressions for Electoral Systems», British Journal of Political Science, 1: 467-477.

MARSHALL, A. W.; OLKIN, I., y PUKELSHEIM, F. (2002): «A majorization comparison of apportionment methods in proportional representation», Social Choice and Welfare, 19 (4): 885-900.

NEWLAND, Robert A. (1982): Comparative Electoral Systems, Arthur McDougall Fund, Londres.

NOHLEN, Dieter (1995): Sistemas Electorales y Partidos Políticos, UNAM, México. 
PALOMARES, A., y RAMíREZ, V. (2003): «Thresholds of the divisor methods», Numerical Algorithms, 34 (2-4): 405415.

PENADÉS, Alberto (1997): «A Critique of Lijphart's “Electoral Systems and Political Systems”», Electoral Studies, 16, 1: 59-71.

- (2000): Los sistemas elementales de Representación, Instituto Juan March, Madrid.

- (2007): «Thresholds and bounds for divisor and quota methods of apportionment», Working Paper, Fundación Juan March, Madrid.

RAE, Douglas W. (1971): The Political Consequences of Electoral Laws, Yale University Press, New Haven (2..$^{\text {a }}$ ed.).

RAE, D.; HANBY, V., y LOOSEMORE, J. (1971): «Thresholds of Representation and Thresholds of Exclusion. An Analytic Note On Electoral Systems», Comparative Political Studies, 4: 479-488.

RAMíREZ, V. (1991): Elecciones en democracia parlamentaria: proporcionalidad y distribución de escaños, Proyecto Sur de Ediciones, Granada.

ROKKAN, S. (1968): «Elections. Electoral Systems», en International Encyclopedia of the Social Sciences, CrowellCollier-Macmillan, New York.

RUIZ-RUFINO, Rubén (2005a): Aggregated threshold functions. A characterization of the world electoral systems between 1945-2000, Instituto Juan March, Centro de Estudios Avanzados en Ciencias Sociales, Madrid.

- (2005b): «La caracterización de los sistemas electorales. Una aplicación de las funciones de agregación de umbrales en las nuevas democracias de Europa y América Latina», Revista Española de Ciencia Política, 13: 89-118.

SCHUSTER, K.; PUKELSHEIM, F.; DRTON, M., y DRAPER, N. R. (2003): «Seat biases of apportionment methods for proportional representation», Electoral Studies, 22 (4): 651-676.

SCHWINGENSCHLOGL, U., y DRTON, M. (2004): «Seat allocation distributions and seat biases of stationary apportionment methods for proportional representation», Metrika, 60 (2): 191-202.

TAAGEPERA, Rein (1989): «Empirical Thresholds of Representation», Electoral Studies, 8 (1): 105-116.

- (1998): «Effective Magnitude and Effective Threshold», Electoral Studies, 17 (4): 393-404.

- (2002): «Nationwide threshold of representation», Electoral Studies, 21: 383-401.

TAAGEPERA, R., y LAAKSO, M. (1980): «Proportionality profiles of west European electoral systems», European Journal of Political Research, 8 (4): 423-446.

TAAGEPERA, R., y SHUGART, M. S. (1989): Seats and Votes. The Effects and Determinants of Electoral Systems, New Haven.

URDÁNOZ, Jorge (2006a): Fórmulas Electorales y Representación Proporcional, Universidad Pública de Navarra, Pamplona.

- (2006b): «Medición de la desproporcionalidad electoral: una crítica a los Mínimos Cuadrados», Revista Española de Investigaciones Sociológicas, 115: 257-296.

- (2007): “La teoría de los precios: una explicación sistemática para las fórmulas electorales», Revista Española de Ciencia Política, 17: 113-154. 